system, that if all the dust, or meteorites, or conglomerations of particles, whatever they may be, are going the same way, there will be a condition in which we shall get a minimum of collisions, and therefore a minimum of temperature.

The probability, therefore, is that we are not dealing with gas, but with masses of matter in certain regions of which, in consequence of general action, there is greater luminosity given off by the particles of which the nebulæe are composed; in

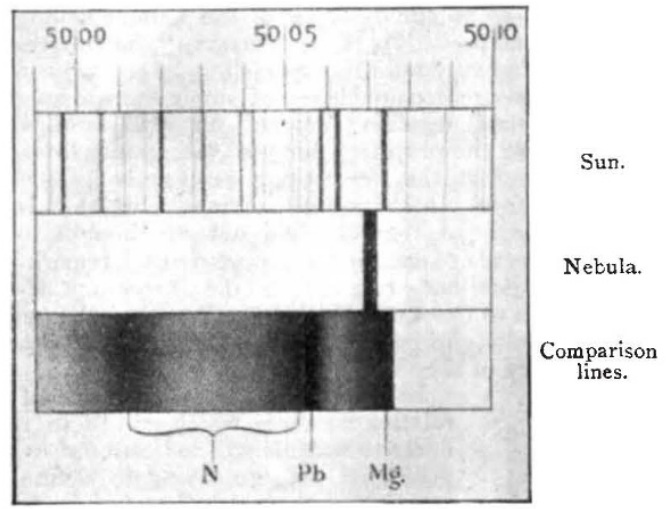

FIG. 20.- Normal position of the chief nebular line, according to Keeler.

other regions where there is less action, we have lower temperature and less light.

If, as was at first imagined, these nebulæ are gases at enormous temperatures, it would be a question of seeing them or not seeing them; there would be no special parts to be picked out at all. But, in the case of those nebulæ to which modern photographic methods have been applied, we find that
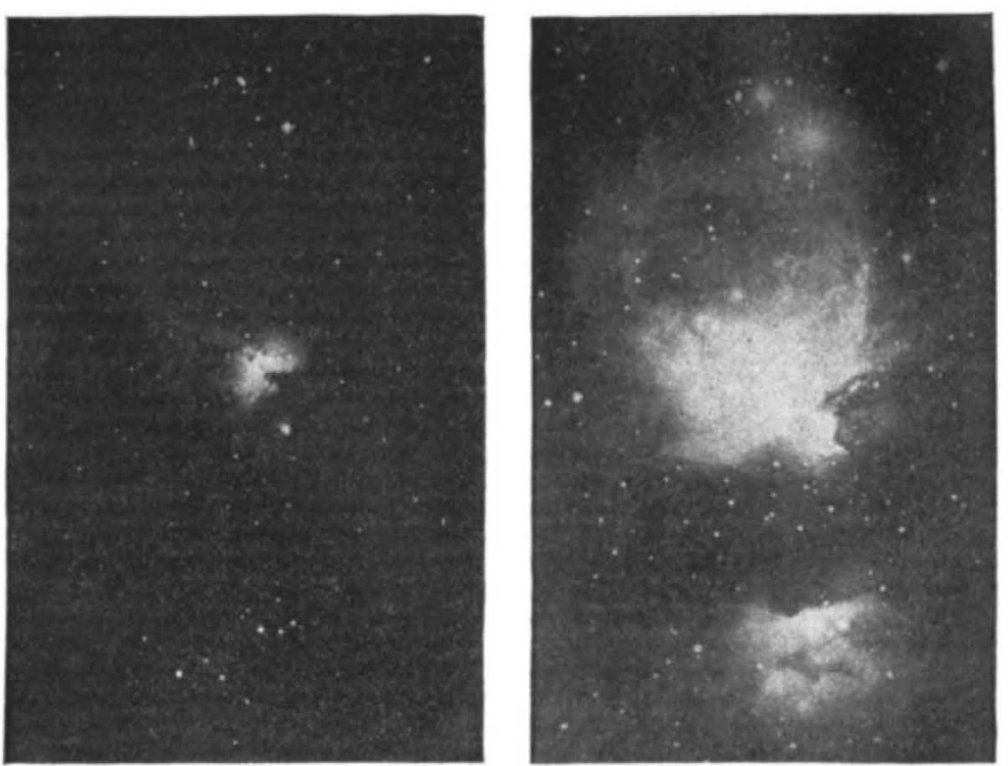

FIG. 21,-Orion nebula photographed with short and long exposures.

the nebula which we see ordinarily in our telescopes is only a very, very small fraction of the real nebula as it really exists, when we can get at it under the best possible observing conditions. Many of you, I hope, have seen the nebula of Orion in an ordinary telescope. Here it is as it has been photographed by means of a telescope powerful enough to give us the brighter portions. Here is anotner photograph of the nebula exactly on the same scale, in which the nebula that we usually see occupie; only a very small portion; the only difference between the two photographs is that one has been exposed for a very long time to enable us to fix and to study the very dim reproduction of certain parts of it, whilst the first one was exposed only for a short time, in order that we might dwell effectively on that part only of the nebula which is generally visible to the human eye with an ordinary telescope. (Fig. 2.) If we were dealing with incandescent gas, the incandescent gas ought to leave off sud. denly; but all round this nebula, where there appears to be nothing at all, the longer exposure brings before us other portions of the nebula just as rich in details, just as exquisite in their variety and tone as those ordinarily seen with the naked eye. Such a condition as that cannot be brought about by a mere homogeneous mass of gas at high temperature, but we can explain it quite easily by assuming that in such a nebula as that we are dealing with, the luminosity is brought about by disturbances, these disturbances giving rise to collisions among the particles which are apt to collide and give out luminosity. The nearer they are to the centre of gravity of the swarm, the greater will be their chance of collision, and the greater will be the luminosity of their central portion.

Still another consideration. Astronomers, since the time of Rutherfurd, who was the first to begin stellar spectroscopic work in the United States, between 1860 and 1870 , have established many different classes of stars as defined by the chemical substances of which their atmospheres seem to be composed, so far as spectroscopic observations enable us to determine their composition. One group of stars is remarkable for the presence of hydrogen in enormous quantities; we assume that because the lines of hydrogen are inordinately thick. In another we get not so much hydrogen, although it is still there, but the predominant substance is iron. In other stars we get little hydrogen, if any, apparently no iron, but carbon in enormous quantities, and again there is another substance, the quantity of which varies enormously, and that is calcium. Now, if stars contain all these different substances, and if they represent epochs of evolution, they must be produced from something which actually or potentially contained these substances, so that there again you get a considerable argument in favour of the chemical complexity of the nebulæ.

Finally, we reach the second point. It is now generally conceded that the first stage in the development of cosmical bodies is not a hot gas, but a swarm of cold meteorites. From the point of view of evolution, keeping well in touch with the laws of thermodynamics, the nebulæ must begin cool if they are to develop into hot stars. J. NORMAN LOCKYER. (7o le continuea).

NEW COMPOUNDS OF PHOSPHORUS, NITROGEN, AND CHLORINE.

A SERIES of new compounds of phosphorus, nitrogen, and chlorine, and iikewise a series of acids derived from them, have been discovered by Mr. H. N. Stokes, and an account of them is contributed to the American Chemical fournal. A familiar compound of the three elements in question, the chlorophos. phuret of nitrogen, discovered by Liebig in 1832 , has been the subject of frequent study, and its nature has comparatively recently been very fully demonstrated by Dr. Gladstone. It has been shown, from vapour density determinations, that this remarkably stable compound, which may be distilled in steam and boiled with acids and alkalies without appreciable change, possesses the molecular composition $\mathrm{P}_{3} \mathrm{~N}_{3} \mathrm{Cl}_{6}$. Mr. Stokes now shows that this substance is only one of a homologous series of compounds having the general formula $\left(\mathrm{PNCl}_{2}\right) \mathrm{n}$, and that these are the chlorides of a series of acids $\left(\mathrm{PNO}_{2} \mathrm{H}_{2}\right) \mathrm{n}$, which he terms metaphosphimic acids. The second member of the series, $\left(\mathrm{PNCl}_{2}\right)_{4}$, has been isolated from the product of $\mathrm{Dr}$ Gladstone's reaction for the preparation of $\left(\mathrm{PNCl}_{2}\right)_{3}$, that be

NO. I 329 , VOL. $5 \mathrm{I}]$ 
tween phosphorus pentachloride and ammonium chloride; it is a substance almost as stable as the triple compound, and yields on saponification the acid $\left(\mathrm{PNO}_{2} \mathrm{H}_{2}\right)_{4}$, which is likewise a stable body. Moreover, the acid corresponding to the triple compound has been isolated, and also a higher chloride $\left(\mathrm{PNCl}_{2}\right) \mathbf{x}$ of an oily character, and whose molecular weight has not yet been ascertained. In describing his repetition of Dr. Gladstone's work, Mr. Stokes incidentally mentions the interesting circumstance that the triple compound readily forms enormous crystals, well-developed prisms several inches long and of considerable thickness being frequently deposited from benzene, and indeed their size appears only to be limited by that of the containing vessel and the bulk of solution. These crystals melt at II $4^{\circ}$. The quadruple compound melts at $123^{\circ} \cdot 5$, and boils at the normal pressure at $256^{\circ} \cdot 5$. It crystallises well in colourless prisms, which are usually much smaller than those of the triple compound, and exhibit great tendency to develop an a cicular character. It is less soluble in alcohol and benzene than the latter compound; it may be recrystallised from glacial acetic acid, but it exhibits a great aversion for water, not being wet by it, and consequently the crystals float on water. It dissolves in hot concentrated oil of vitriol, but upon boiling most of it sublimes unchanged, an evidence of its great stability. Its vapour is endowed with a pleasant aromatic odour, but inhalation of more than traces is followed in two or three hours by alarming difficulty in breathing and persistent irritation of the air passages. Its vapour density was determined in an atmosphere of hydrogen, and indicated the quadruple formula. Even boiling water exerts only a very slight action upon it; but a smooth decomposition is effected by dissolving in ether, and repeatedly agitating with water. The acid produced is deposited from the water in crystals having the composition $\left(\mathrm{PNO}_{2} \mathrm{H}_{2}\right)_{4}+2 \mathrm{H}_{2} \mathrm{O}$. This interesting acid readily decomposes soluble chlorides, nitrates, and sulphates, forming three series of salts, in which respectively one-fourth, one-half, and all the hydrogen is replaced by the metal. The free acid is so highly stable that it may be boiled for hours with nitric acid or aqua regia without decomposition. Further details concerning it, and the other compounds isolated, will shortly be published.

\section{BIOLOGICAL WORK ON THE ILLINOIS RIVER.}

I LLINOIS possesses a good Laboratory of Natural History, in which Prof. S. A. Forbes, with a number of assistant entomologists and zoologists, carry on investigations of value to science and the State. A report on the work of the Laboratory during the past two years has recently been issue d. To us the points of special interest with which it deals are (I) the establishment, in 1894, of a biological station for the continuous investigation of the aquatic life of the Illinois River, and its dependent waters, near Havana; and (2) an elaborate expenimental research carried on during the past year to determine means for the destruction of the chinch bug, and especially for the dissemination of the contagious diseases of that insect. This investigation was undertaken by the Laboratory staff, with the co-operation of the State Agricultural Experiment Station.

We have already noted the establishment of the biological station (NATURE, vol. 1. p. 275, 1894), but as it is the first inland aquatic biological station in America, manned and equipped for continuous investigation, the following further details are interesting. By the kindness of Prof. Forbes, we are able to illustrate the description with two of the fifteen fine process plates contained in his report.

The Station was opened just a year ago. Its general objects are to provide additional facilities and resources for the study of the natural history survey of the State, now being carried on by the State Laboratory of Natural History; to contribute to a thorough scientific knowledge of the whole system of life existing in the waters of this State, with a view to economic as well as educational applications, and especially with reference to the improvement of fish culture and to the prevention of a progressive pollution of Illinois streams and lakes; to occupy a rich and promising field of original biological investigation hitherto largely overlooked or neglected, not only in America but throughout the world; and to increase the resources of the zoological and botanical depart. ments of the University of Illinois, by providing means and facilities for special lines of both graduate and undergraduate work and study, for those taking major courses in these depart. ments.

The Station differs from most of the small number of similar stations, thus far established in the United States, in the fact that its main object is investigation instead of instruction, the latter being a secondary, and at present an incidental, object only. It has for its field the entire system of life in the Illinois River and connected lakes and other adjacent waters, and it is Prof. Forbes' intention to extend the work as rapidly as possible to the Mississippi River-system, thus making a beginning on a comprehensive work in the general field of the aquatic life of the Mississippi Valley, in all its relations, scientific and economic.

The special subject which Prof. Forbes fixed upon as the point of direction towards which all the studies shall tend, is the effect, on the aquatic plant and animal life of a region, produced by the periodical overflow and gradual recession of the waters of great rivers, phenomena of which the Illinois and Mississippi rivers afford excellent and strongly marked examples. This field of research is entirely fresh, and at the same time is highly interesting and important.

As an incidental, but by no means unimportant, result of the work, material will be accumulated for a comparison of the chemical and biological conditions of the waters of the Illinois River, at the present time, and after the opening of the Chicago drainage canal.

The practical importance of the undertaking, as affording the only sound basis for a scientific fish culture, will be fully recognised by all who are seeking to apply scientific methods of investigation to economic problems.

It is pointed out that the Station will also serve as a centre of interest and activity for University students engaged on zoological and botanical subjects. Not many years ago biological instruction in American colleges was mostly derived from books; of late it has been largely obtained in laboratories instead; but Prof. Forbes believes that the mere book-worm is hardly narrower and more mechanical than the mere laboratory grub. Both have suffered, and almost equally, from a lack of opportunity to study nature alive. One knows about as much as the other of the real aspect of living nature, and of the ways in which living things limit and determine each others' activities and characters, or in which all are determined by the inorganic environment.

Means are provided by which students, and intending teachers of biology, may be given a wider knowledge of their subject, and where they may investigate experimentally the problems of mutual influences and relationship in the living world.

Possibly still more important is the opportunity which the Station will offer, when permanently established and fairly well developed, to the independent student and investigator, zoological or botanical, who may desire to pursue his studies in the field covered by the operations. It is a part of the plan of organisation and equipment to receive and assist in every practicable way advanced students and investigators of this description, from whatever place they may come.

Havana was selected as the site of the Station because of several unique advantages offered by that locality. Streams and lakes illustrating practically all the typical Illinois River situations are to be found there, convenient of access from a central point, and from each other. An extensive sandy bluff, commonly well shaded and oozing spring water at its foot, borders the river bottom on the east, and introduces several un. usual features of interest to the œcologist, besides affording a clean and hard shore to work from, dry, shady, and well-drained camping ground, and an abundance of very pure cold water at all times of the year.

A "cabin boat" was used as a field headquarters, and stationed on Quiver Lake, two and a half miles above Havana. It carried the seines, sounding lines, aërial and aquatic ther. mometers, dredges, surface nets, Birge nets, insect nets, plankton apparatus, and other collecting equipment, together with microscopes, reagents, a small working library, a large number of special breeding cages for rearing aquatic insects, and a few small aquaria. This boat was provided with sleeping accommodation for four men, and with a well-furnished kitchen.

The greater part of the field work was done on seven regular stations, visited periodically throughout the year; two on the

NO. I 329 , VOL. 5 I] 